\title{
Synchronous multiple lung cancers presenting as multifocal pure ground glass nodules: are whole-body positron emission tomography/computed tomography and brain enhanced magnetic resonance imaging necessary?
}

\author{
Meng Li ${ }^{1}$, Yuan $\mathrm{Wan}^{1}$, Li Zhang ${ }^{1}$, Li-Na Zhou ${ }^{1}$, Zhuo Shi ${ }^{1}$, Rui Zhang ${ }^{1}$, Yan-Lei Hou ${ }^{1}$, Ning Wu ${ }^{1,2}$ \\ ${ }^{1}$ Department of Diagnostic Radiology, ${ }^{2}$ PET-CT Center, National Cancer Center/National Clinical Research Center for Cancer/Cancer Hospital, \\ Chinese Academy of Medical Sciences and Peking Union Medical College, Beijing 100021, China \\ Contributions: (I) Conception and design: M Li, LN Zhou, N Wu; (II) Administrative support: N Wu; (III) Provision of study materials or patients: \\ M Li, Z Shi, R Zhang, YL Hou; (IV) Collection and assembly of data: M Li, Y Wan; (V) Data analysis and interpretation: M Li, L Zhang; (VI) \\ Manuscript writing: All authors; (VII) Final approval of manuscript: All authors. \\ Correspondence to: Ning Wu, MD. Department of Diagnostic Radiology, PET-CT Center, National Cancer Center/National Clinical Research \\ Center for Cancer/Cancer Hospital, Chinese Academy of Medical Sciences and Peking Union Medical College, No. 17 Panjiayuan Nanli Chaoyang \\ District, Beijing 100021, China. Email: cjr.wuning@vip.163.com.
}

Background: Multifocal ground glass nodules (GGNs) represent a special radiological pattern indicative of synchronous multiple lung cancers (SMLCs), especially adenocarcinoma. However, the necessity of performing whole-body positron emission tomography/computed tomography (PET-CT) scanning and brain enhanced magnetic resonance imaging (MRI) as a staging workup for multifocal pure GGN (pGGN) patients remains unclear. The purpose of this study was to determine the utility of these two imaging scans for patients with multifocal pGGNs.

Methods: This retrospective study was reviewed and approved by the ethics committee of the Cancer Hospital of the Chinese Academy of Medical Sciences. The study cohort was retrospectively selected from patients with multifocal pGGNs who underwent whole-body PET-CT examinations and/or brain enhanced MRIs between January 2010 and February 2019 at our institution. The additional value of the two exams for detecting nodal and distant metastases was evaluated.

Results: In total, 73 patients (male-to-female ratio, 20:53; median age, 57 years) with multifocal pGGNs who underwent whole-body PET-CT (55 patients) and/or brain enhanced MRI (25 patients) were enrolled. No clearly metastatic lesions were detected. Among the enrolled patients, 53 (128 pGGNs) underwent complete surgical resection. All pGGNs were adenocarcinomas and/or preneoplasias, and no lymph node metastases were found on final pathology. Whole-body PET-CT and brain enhanced MRI added no definite benefit compared with chest CT alone before surgery.

Conclusions: Whole-body PET-CT scans and brain enhanced MRIs are not necessary for patients with multifocal pGGNs.

Keywords: Lung cancer; magnetic resonance imaging (MRI); multifocal ground glass nodules (multifocal GGNs); positron emission tomography/computed tomography (PET-CT)

Submitted May 09, 2019. Accepted for publication Aug 29, 2019.

doi: $10.21037 /$ tlcr.2019.09.10

View this article at: http://dx.doi.org/10.21037/tlcr.2019.09.10 


\section{Introduction}

Although the incidence of synchronous multiple lung cancers (SMLCs) has been reported to account for $0.2-20 \%$ of all lung cancers, SMLCs appear to be increasing in global frequency (1-5). This may be a result of the prevalence of low-dose computed tomography (LDCT) screening and high-resolution CT (HRCT) scanning, especially for the detection of ground glass nodules (GGNs) in the lung that are not visible on conventional radiographs (6). However, for decades, patients with lung cancers that harbor multiple pulmonary sites of involvement have been challenging to classify because of an inability to distinguish between independent primary tumors and intrapulmonary metastases (7). Additionally, several patterns of radiological presentation are associated with SMLC. To provide better clarity, the 8th edition of the new International Association for the Study of Lung Cancer (IASLC) staging system proposed well-defined criteria for categorizing multiple lung cancers into four patterns of presentation, i.e., second primary lung cancer, separate tumor nodule, multifocal GGNs, and diffuse pneumonic-type lung cancer $(8,9)$.

The 2019 National Comprehensive Cancer Network (NCCN) guidelines recommend positron emission and computed tomography (PET-CT) scans and brain enhanced magnetic resonance imaging (MRI) for multiple lung cancers (10), without mentioning any patterns. [18F] Fluorodeoxyglucose $\left({ }^{18} \mathrm{~F}-\mathrm{FDG}\right)$ PET-CT is recommended for the clinical or preoperative evaluation of mediastinal and distant metastases in patients with lung cancer $(11,12)$. Due to the shortcomings of PET-CT for brain imaging, brain enhanced MRI is the best method of evaluating neurologic metastasis (13).

Meanwhile, as a radiological pattern of SMLCs, multifocal GGNs are becoming more frequent, although the incidence has not been quantified $(14,15)$. However, further uncertainty regarding the necessity of PET-CT and brain MRI is increasing in patients with SMLCs presenting with multifocal pure GGNs (pGGNs) in our daily clinical practice. Therefore, we retrospectively evaluated the usefulness and necessity of the routine use of whole-body PET-CTs and brain MRIs for the detection of mediastinal and distant metastases in patients with multifocal predominant pGGNs and thus determined whether these imaging procedures are mandatory as either preoperative evaluations or follow-up examinations.

\section{Methods}

This retrospective study was reviewed and approved by the ethics committee of the Cancer Hospital of the Chinese Academy of Medical Sciences (No. NCC2013RE-054), which waived the requirement for informed consent due to the retrospective and anonymous data analysis.

\section{Patient selection}

The study cohort was retrospectively selected from patients with multifocal pGGNs who underwent whole-body PETCT examination and/or brain enhanced MRI between January 2010 and February 2019 at our institution. The pGGN was defined as a hazy increased opacity in the lung, with preserved bronchial and vascular margins (16-19). The selection criteria for this study were available thin-section chest CT scans that indicated more than two multifocal pGGNs according to the above definition, with preserved bronchial and vascular margins on lung window images and with no or barely identifiable soft-tissue attenuation within the lesion on mediastinal window images. The CT diagnosis was of multifocal pGGNs suspected of being lung cancer. Two radiologists (with 11 and 10 years of experience in chest CT) evaluated the images and came to a consensus regarding patient enrollment.

\section{Image acquisition and analysis}

Whole-body PET-CT scans and brain MRIs were performed using our standard protocols. For the PETCT examinations, after a normal blood glucose level in the peripheral blood was ensured $(\leq 8 \mathrm{mmol} / \mathrm{L})$, patients received an intravenous injection of ${ }^{18} \mathrm{~F}-\mathrm{FDG}$ at $3.70-4.44 \mathrm{MBq} / \mathrm{kg}$ and then rested for 50-70 min before undergoing the scan. Image acquisition was performed with an integrated PETCT device (GE Discovery ST 16) that consisted of a PET scanner and a 16-slice CT scanner. The whole body was scanned from the head to the groin. The CT scan used a standardized protocol that involved $120 \mathrm{kV}, 150 \mathrm{~mA}$, 0.8 seconds per tube rotation, and a section thickness of $3.75 \mathrm{~mm}$. No intravenous contrast material was administered. Immediately after the CT scan, PET was performed in an identical transverse field of view with a section thickness of $3.27 \mathrm{~mm}, 3$ minutes per table position and three-dimensional acquisitions. Patients were asked to 


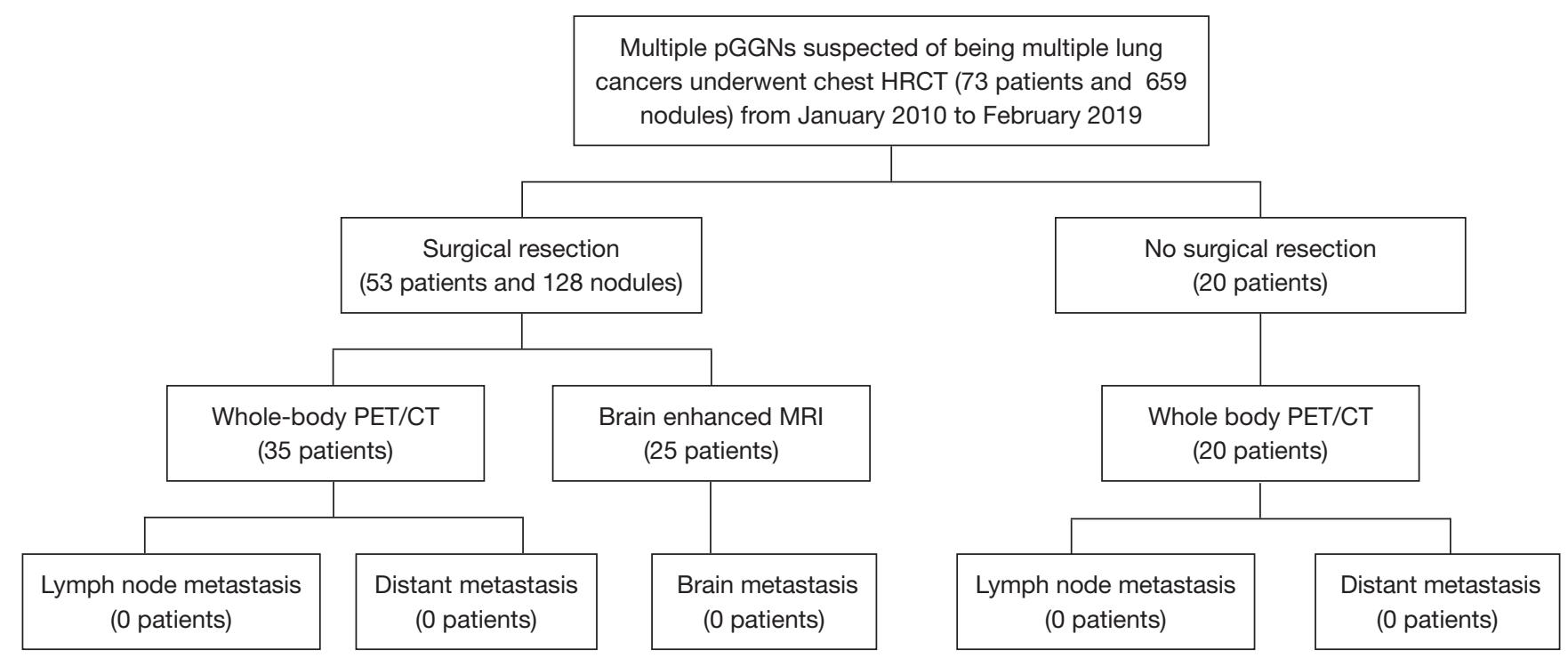

Figure 1 Flow chart of patient enrollment and staging workup for patients with multiple pure ground glass nodules suspected of being synchronous multiple lung cancers.

maintain normal shallow respiration during the acquisition of the image. Coregistered images were displayed using software (Xeleris) that allowed image fusion and analysis. All patients underwent a breath-hold thoracic spiral CT scan with $120 \mathrm{kV}, 205 \mathrm{~mA}, 0.8$ seconds per tube rotation, and a thickness of $5 \mathrm{~mm}$ after the PET-CT scan. Images were reconstructed from a thickness of $1.25 \mathrm{~mm}$ and a space of $1 \mathrm{~mm}$.

Brain enhanced MRI scans were obtained with 1.5 or 3.0 T MRI scanners (GE) and included axial FSE T2WI/ FLAIR (TR, 8,000 ms; TE, $130 \mathrm{~ms}$; NEX, 1; slice thickness, $5 \mathrm{~mm}$ and slice space, $1 \mathrm{~mm}$ ) and axial and sagittal FSE T1WI scans with intravenous injection of Gd-DTPA contrast (TR, $420 \mathrm{~ms}$; TE, $20 \mathrm{~ms}$; NEX, 1; slice thickness, 5 $\mathrm{mm}$ and slice space, $1 \mathrm{~mm}$ ).

\section{Surgical procedure and pathologic stage}

If surgical resection was performed, the pathological diagnosis and stage were recorded. The histological classification of adenocarcinoma followed the IASLC/ American Thoracic Society/European Respiratory Society classification (20). The mutation statuses of the epidermal growth factor receptor (EGFR) and Kirsten rat sarcoma viral oncogene homolog (KRAS) genes examined by molecular pathological analyses were recorded if available.

\section{Results}

\section{Characteristics of the study patients}

Seventy-three patients (male-to-female ratio, 20:53; median age, 57 years) with multifocal pGGNs were enrolled in the analysis (Figure 1). In total, the 73 patients had 659 GGNs, and the mean lesion size was $1.6 \pm 0.9 \mathrm{~cm}$. The clinical and radiological findings for the total study population are summarized in Table 1.

\section{Additional value of whole-body PET-CT and brain enhanced MRI for nodal staging and distant metastasis}

Whole-body ${ }^{18} \mathrm{~F}$-FDG PET-CT scans and/or brain MRIs were performed for all of the enrolled patients. Of these patients, imaging data were available for 55 who underwent whole-body PET-CT and 25 who underwent brain enhanced MRI. Seven patients underwent both whole-body PET-CT and brain enhanced MRI. No definitely metastatic lesions were detected in the 55 patients who underwent whole-body PET-CT, and no brain metastasis was found on brain enhanced MRIs in any of the 25 patients (Figures 1,2).

\section{Surgical procedure and pathological outcome}

Among the enrolled patients, 53 (128 pGGNs) underwent 
Table 1 Clinical and radiological characteristics of all 73 patients

\begin{tabular}{|c|c|}
\hline Variable/characteristic & $\begin{array}{c}\text { Results/No. of } \\
\text { patients (\%) }\end{array}$ \\
\hline Median age, years & 57 (range, 37-75) \\
\hline \multicolumn{2}{|l|}{ Sex } \\
\hline Male & $20(27.4)$ \\
\hline Female & $53(72.6)$ \\
\hline \multicolumn{2}{|l|}{ History of smoking } \\
\hline Smoker & $12(16.4)$ \\
\hline Nonsmoker & $61(83.6)$ \\
\hline \multicolumn{2}{|l|}{ Initial symptoms } \\
\hline Asymptomatic & $57(78.1)$ \\
\hline Cough & $16(21.9)$ \\
\hline Total No. of pGGNs & 659 \\
\hline \multicolumn{2}{|l|}{ No. of pGGNs per patient } \\
\hline $1-5$ & $44(60.3)$ \\
\hline $6-10$ & $14(19.2)$ \\
\hline $11-20$ & $9(12.3)$ \\
\hline$>20$ & $6(8.2)$ \\
\hline Average tumor size of main lesion on $\mathrm{CT}, \mathrm{cm}$ & $1.6 \pm 0.9$ \\
\hline \multicolumn{2}{|l|}{ Tumor size of main pGGN lesion on CT } \\
\hline$<1 \mathrm{~cm}$ & $18(24.7)$ \\
\hline $1-1.9 \mathrm{~cm}$ & $33(45.2)$ \\
\hline $2-2.9 \mathrm{~cm}$ & $14(19.2)$ \\
\hline$>3 \mathrm{~cm}$ & $8(11.0)$ \\
\hline Average $\mathrm{SUV}_{\max }$ of main lesion on PET-CT & 0.7 \\
\hline
\end{tabular}

complete surgical resection with curative intent and all had pathology-proven adenocarcinomas and/or preneoplasias, including 70 adenocarcinomas, 9 minimally invasive adenocarcinomas (MIAs) and 49 atypical adenomatous hyperplasias (AAHs)/adenocarcinomas in situ (AISs). None of the tumors displayed lymph node involvement or lymphatic invasion, and no pleural involvement was observed in the pathological examinations. Twenty-six patients (28 pGGNs) underwent molecular pathological examinations, and EGFR mutations were found in 18 pGGNs, including 1 EGFR18, 7 EGFR19, 1 EGFR20, and
9 EGFR21 exon mutations. With regard to these mutations, two paired GGNs (EGFR18/20 and EGFR19/21) were in the same patient. The KRAS mutation was found in only 1 patient (Table 2).

\section{Discussion}

We performed a retrospective observational study to evaluate the additional benefit of whole-body PET-CT and brain enhanced MRI compared to chest CT alone in patients with multifocal pGGNs. In this study, no definite mediastinal or distant metastases were observed in the 73 enrolled patients, although false-positive uptake usually occurred in the PET-CT studies. There were also no cases of lymph node metastasis in the final pathology of any of the patients who underwent operations. Although the necessity of these two imaging procedures in patients with multifocal pGGNs lacks supporting evidence and scientific consensus, these procedures are usually performed, probably due to the NCCN guidelines for SMLCs, especially before surgery. To the best of our knowledge, this is the first study on the necessity of whole-body PET-CT and brain enhanced MRI in patients with synchronous multifocal pGGNs. We concluded that whole-body PET-CT and brain enhanced MRI add little value to the staging of multifocal pGGNs compared to chest CT alone. In contrast with the NCCN guidelines for SMLCs, we conclude that these additional imaging procedures are unnecessary. Given the frequency with which pGGNs are encountered in daily clinical practice, our study can facilitate cost savings and reduce unnecessary radiation.

On CT images, lung nodules are classified as solid nodules (SNs), part-solid nodules (PSNs) or mixed GGNs (mGGNs), and nonsolid nodules (NSNs) or pGGN; in addition to pGGN/NSN, mGGN/PSN is defined as a combination of ground glass and solid attenuation, which obscures the underlying lung architecture on CT. pGGN/ NSN and mGGN/PSN have both been referred to as subsolid nodules (SSNs) (16-19). In our study, we identified pGGNs in thin chest CT images (thickness of $1.25 \mathrm{~mm}$, space of $1.0 \mathrm{~cm}$ ). Every PET-CT scan has an additional breath-hold high resolution CT scan, so the observation of pGGNs is reliable.

Persistent focal GGNs on follow-up CT scans are associated with early-stage lung adenocarcinomas (21). According to the new lung adenocarcinoma classification system, a less invasive lepidic adenocarcinoma cell growth 

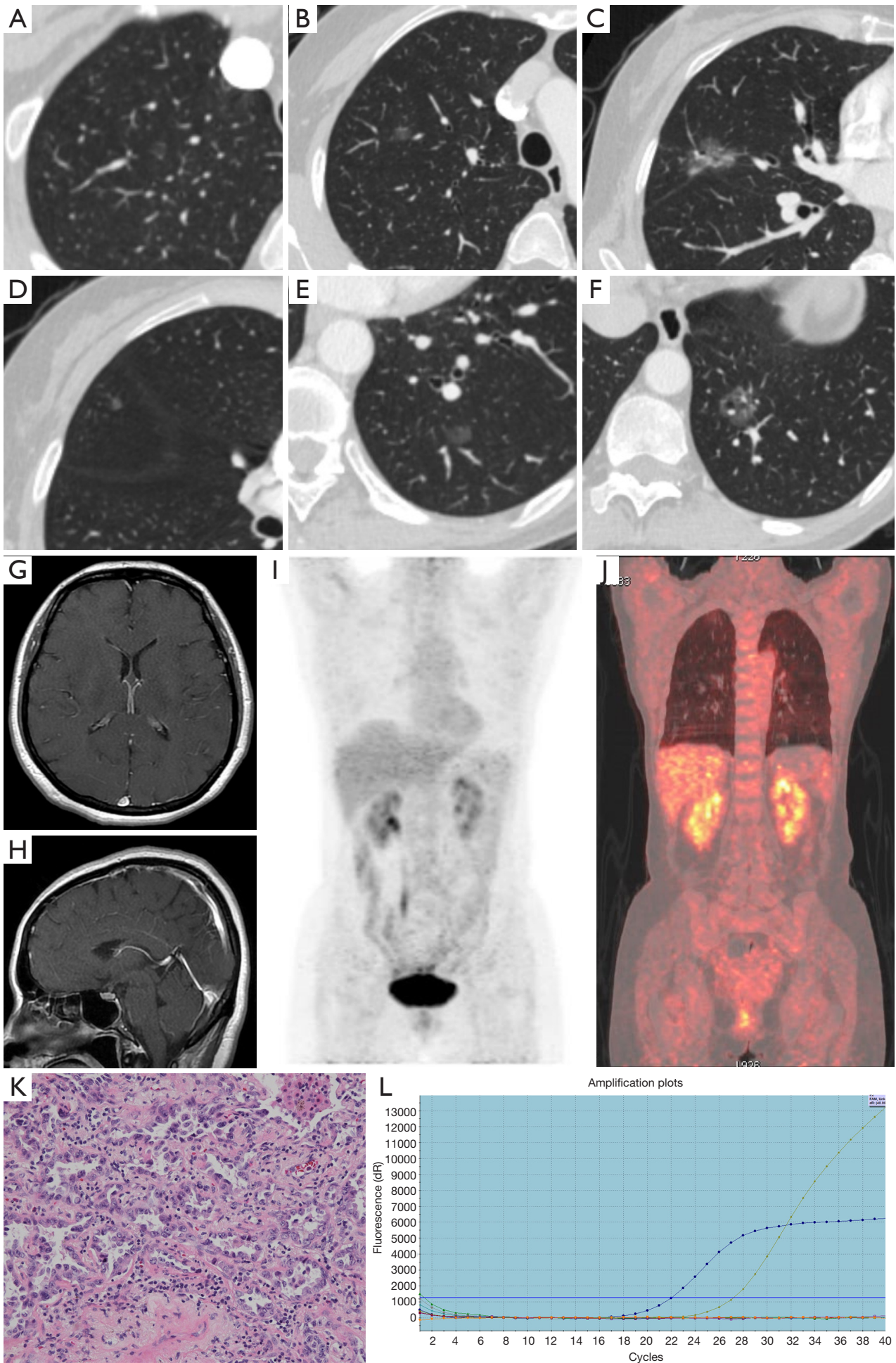

Figure 2 A 64-year-old female. (A,B,C,D,E,F) CT images show six pure ground glass nodules (pGGNs) in both lungs. (G,H) Brain MRI images show no brain metastasis. (I) A maximum intensity projection image of whole-body PET and (J) an integrated PET-CT image show no mediastinal and distant metastasis. (K) The histopathology of the main lesion in the right upper lobe after surgery shows adenocarcinoma (HE, $\times 200)$, and other three pGGNs in the right lung resected as well were adenocarcinomas in situ according to the pathological results. (L) The molecular pathological result of the main lesion shows EGFR21 mutations. 
Table 2 Surgical procedures and pathological characteristics of patients who underwent operations

\begin{tabular}{|c|c|}
\hline Variable/characteristic & $\begin{array}{c}\text { Results/No. of } \\
\text { patients (\%) }\end{array}$ \\
\hline Surgical procedure patients & $53(72.6)$ \\
\hline pGGNs resected & $128(19.4)$ \\
\hline \multicolumn{2}{|c|}{ Pathological lymph nodal metastasis } \\
\hline No $\left(\mathrm{N}_{0}\right)$ & $73[100]$ \\
\hline Yes $\left(\mathrm{N}_{1-2}\right)$ & $0(0)$ \\
\hline \multicolumn{2}{|l|}{ Histological type (128 pGGNs) } \\
\hline $\mathrm{AAH}$ and AIS & 49 (38.3) \\
\hline MIA & $9(7.0)$ \\
\hline Adenocarcinoma & $70(54.7)$ \\
\hline \multicolumn{2}{|c|}{ Molecular pathology (28 pGGNs) } \\
\hline None & $9(32.1)$ \\
\hline EGFR & $18(64.3)$ \\
\hline EGFR18 & 1 \\
\hline EGFR19 & 7 \\
\hline EGFR20 & 1 \\
\hline EGFR21 & 9 \\
\hline KRAS & $1(3.6)$ \\
\hline
\end{tabular}

MIA, minimally invasive adenocarcinoma; $A A H$, atypical adenomatous hyperplasia; AIS, adenocarcinoma in situ; EGFR, epidermal growth factor receptor; KRAS, Kirsten rat sarcoma viral oncogene homolog; pGGNs, pure ground glass nodules.

pattern observed on microscopy permits aeration of the alveoli and results in this characteristic appearance on CT (22). In our study, all the resected pGGNs were adenocarcinomas or preneoplasia, including AAH/AIS, MIA. Adenocarcinoma is the most common histological form of lung cancer and is associated with mutations in three common oncogenes: EGFR, anaplastic lymphoma kinase (ALK), and KRAS. Radiogenomic studies demonstrated that the GGN rate was associated with EGFR mutations in lung adenocarcinoma $(23,24)$. In our study, 28 resected pGGNs were subjected to molecular pathological examination, and the EGFR mutation rate was $64.3 \%$ (18/28).

The cause of GGNs is a major issue that remains unresolved. There are several differences between lung cancer with GGNs and typical lung cancer. Unlike typical lung cancers, GGNs are not associated with smoking, a major cause of lung cancer, as most patients are nonsmokers. GGNs occur at a relatively young age and have a very indolent course. Moreover, they develop in the peripheral portion of the lungs, and many of them have a multifocal origin. A previous study suggested genetic susceptibility, household air pollution attributed to the burning of solid fuel for heating and cooking, and cooking fumes as causative agents in China (25). In our study, females $(72.6 \%, 53 / 73)$ and nonsmokers $(83.6 \%, 61 / 73)$ accounted for the majority of the cohort.

Like other SMLCs, multiple GGNs used to present a clinical dilemma regarding whether they represent intrapulmonary metastases from a single primary cancer or independent multiple primary lung cancers (MPLCs) from different origins $(26,27)$; this dilemma was not resolved by pathological evaluation because they are always of the same histological type. According to the traditional definition, MPLCs with the same pathologies must meet the following criteria: the origin was a carcinoma in situ, with no lymph node involvement in the common lymphatic pathways and no extrathoracic metastasis $(28,29)$. In this study, we found no metastasis on PET-CT imaging, which serves as imaging evidence verifying that the multiple GGNs are MPLCs with a multifocal origin. In statements from the Fleischner Society and IASLC, GGNs are considered MPLCs in the early stage of tumorigenesis $(30,31)$.

In addition to attempting to classify multiple GGNs based on pathological presentation, the incorporation of genetic or molecular profiles into the differential diagnosis has recently been adopted and strongly supports that they are all independent primary tumors $(32,33)$. In this study, two pairs of GGNs that were each resected from a single patient were subjected to molecular pathological examinations, and the locations of the EGFR exon mutation were different (EGFR18 and 20 in one patient, EGFR19 and 21 in the other patient), which may confirm the different clonal origins of multiple GGNs.

However, the possibility of intrapulmonary metastasis of small multifocal GGNs has been reported. Li et al. (34) analyzed the clonal origins of multiple GGNs with matched samples in two patients using exome sequencing and found that two GGNs in each patient shared multiple nonsynonymous and synonymous mutations, which strongly suggested intrapulmonary metastasis, and the remaining GGNs showed different clonal origins. This phenomenon may be due to the spread of the tumors through air spaces, a new metastatic model of lung cancer and a concept that has been gradually accepted $(35,36)$. Whether the metastasis of 
GGONs influences the prognosis remains to be explored. However, more cases are needed for verification in addition to this single study.

Many studies have demonstrated that GGNs (both isolated and multifocal tumors) on CT or lepidic components on microscopy exhibit rather indolent behavior and are associated with an improved prognosis (37-39). According to the multifocal origin and the rarity of regional lymph nodal metastasis, which was also demonstrated in our study, surgery for multifocal dominant GGNs by lobectomy or, preferably, limited resection is recommended, and then close follow-up of any remaining GGNs would be the optimal strategy $(40,41)$. However, the complete analysis of genetic mutations using next-generation sequencing enables us to easily evaluate tumor clonality by performing wholegenome, whole-exome, or targeted gene sequencing $(42,43)$, and it will be necessary for the management of patients in the future.

Our study has several limitations. First, this study had a relatively small sample size. However, PET-CT is expensive, and a large sample was not readily available. Second, our study was inherently limited by its retrospective design and may have been subject to selection bias. Therefore, further prospective studies should be performed to evaluate the utility of preoperative PET-CT and brain MRI. Finally, we included only pure and not part-solid GGNs in this study. Part-solid GGNs may have more aggressive clinical courses than do pGGNs and require further investigations.

\section{Conclusions}

In conclusion, whole-body PET-CT and brain enhanced MRI provide no additional information on metastasis in patients with multifocal pGGNs. Therefore, these imaging procedures seem to be unnecessary in routine clinical practice for these patients.

\section{Acknowledgments}

We thank American Journal Experts for language editorial assistance, Lin Li MD for pathological image assistance, and Lv Lv MD for PET-CT image assistance.

Funding: This work was supported by the National Key R\&D Program of China (2017YFC1308700/2017Y FC1308703), the National Natural Science Foundation of China (Grant No. 81771830, 81701692 and 81601494) and the Fundamental Research Funds for the Central Universities (Grant No. 3332018196).

\section{Footnote}

Conflicts of Interest: The authors have no conflicts of interest to declare.

Ethical Statement: This retrospective study was reviewed and approved by the ethics committee of the Cancer Hospital of the Chinese Academy of Medical Sciences (No. NCC2013RE-054), which waived the requirement for informed consent due to the retrospective and anonymous data analysis. The authors are accountable for all aspects of the work in ensuring that questions related to the accuracy or integrity of any part of the work are appropriately investigated and resolved.

\section{References}

1. Mathisen DJ, Jensik RJ, Faber LP, et al. Survival following resection for second and third primary lung cancers. J Thorac Cardiovasc Surg 1984;88:502-10.

2. Ferguson MK, DeMeester TR, DesLauriers J, et al. Diagnosis and management of synchronous lung cancers. J Thorac Cardiovasc Surg 1985;89:378-85.

3. Wang X, Wang M, MacLennan GT, et al. Evidence for common clonal origin of multifocal lung cancers. J Natl Cancer Inst 2009;101:560-70.

4. Gazdar AF, Minna JD. Multifocal lung cancers--clonality vs field cancerization and does it matter? J Natl Cancer Inst 2009;101:541-3.

5. Jung EJ, Lee JH, Jeon K, et al. Treatment outcomes for patients with synchronous multiple primary non-small cell lung cancer. Lung Cancer 2011;73:237-42.

6. Henschke CI, McCauley DI, Yankelevitz DF, et al. Early lung cancer action project: Overall design and findings from baseline screening. Lancet 1999;354:99-105.

7. Yu YC, Huang CS, Huang BS. Separate or intrapulmonary metastasis? J Thorac Dis 2018;10:S3128-30.

8. Detterbeck FC, Nicholson AG, Franklin WA, et al. The IASLC lung cancer staging project: Summary of proposals for revisions of the classification of lung cancers with multiple pulmonary sites of involvement in the forthcoming eighth edition of the TNM classification. J Thorac Oncol 2016;11:639-50.

9. Detterbeck FC, Boffa DJ, Kim AW, et al. The eighth edition lung cancer stage classification. Chest 2017;151:193-203.

10. NCCN. Clinical practice guidelines in oncology: Nonsmall cell lung cancer (Version 2.2019). 2018. Available 
online: https://www.nccn.org/professionals/physician_gls/ pdf/nscl.pdf. Accessed 21, November 2018.

11. De Leyn P, Dooms C, Kuzdzal J, et al. Revised ESTS guidelines for preoperative mediastinal lymph node staging for non-small-cell lung cancer. Eur J Cardiothorac Surg 2014;45:787-98.

12. Rusch VW, Asamura H, Watanabe H, et al. The IASLC lung cancer staging project: a proposal for a new international lymph node map in the forthcoming seventh edition of the TNM classification for lung cancer. J Thorac Oncol 2009;4:568-77.

13. Lee HY, Lee KS, Kim BT, et al. Diagnostic efficacy of PET/CT plus brain MR imaging for detection of extrathoracic metastases in patients with lung adenocarcinoma. J Korean Med Sci 2009;24:1132-8.

14. Detterbeck FC, Homer RJ. Approach to the ground-glass nodule. Clin Chest Med 2011;32:799-810.

15. Shrager JB. Approach to the patient with multiple lung nodules. Thorac Surg Clin 2013;23:257-66.

16. Hansell DM, Bankier AA, MacMahon H, et al. Fleischner society: Glossary of terms for thoracic imaging. Radiology 2008;246:697-722.

17. Godoy MC, Naidich DP. Subsolid pulmonary nodules and the spectrum of peripheral adenocarcinomas of the lung: Recommended interim guidelines for assessment and management. Radiology 2009;253:606-22.

18. Raad RA, Suh J, Harari S, et al. Nodule characterization: Subsolid nodules. Radiol Clin North Am 2014;52:47-67.

19. Truong MT, Ko JP, Rossi SE, et al. Update in the evaluation of the solitary pulmonary nodule. Radiographics 2014;34:1658-79.

20. Travis WD, Brambilla E, Noguchi M, et al. International association for the study of lung cancer/american thoracic society/european respiratory society international multidisciplinary classification of lung adenocarcinoma. J Thorac Oncol 2011;6:244-85.

21. Kim HY, Shim YM, Lee KS, et al. Persistent pulmonary nodular ground-glass opacity at thin-section CT: Histopathologic comparisons. Radiology 2007;245:267-75.

22. Li M, Wu N, Zhang L, et al. Solid component proportion is an important predictor of tumor invasiveness in clinical stage T1N0M0 (cT1N0M0) lung adenocarcinoma. Cancer Imaging 2018;18:18.

23. Liu Y, Kim J, Qu F, et al. CT features associated with epidermal growth factor receptor mutation status in patients with lung adenocarcinoma. Radiology 2016;280:271-80.

24. Li M, Zhang L, Tang W, et al. Identification of epidermal growth factor receptor mutations in pulmonary adenocarcinoma using dual-energy spectral computed tomography. Eur Radiol 2019;29:2989-97.

25. Hosgood HD 3rd, Song M, Hsiung CA, et al. Interactions between household air pollution and GWAS-identified lung cancer susceptibility markers in the Female Lung Cancer Consortium in Asia (FLCCA). Hum Genet 2015;134:333-41.

26. Homer RJ. Pathologists' staging of multiple foci of lung cancer: Poor concordance in absence of dramatic histologic or molecular differences. Am J Clin Pathol 2015;143:701-6.

27. Asamura H. Multiple primary cancers or multiple metastases, that is the question. J Thorac Oncol 2010;5:930-1.

28. Martini N, Melamed MR. Multiple primary lung cancers. J Thorac Cardiovasc Surg 1975;70:606-12.

29. Shen KR, Meyers BF, Larner JM, et al. Special treatment issues in lung cancer: ACCP evidencebased clinical practice guidelines ( 2 nd edition). Chest 2007;132:290S-305S.

30. Naidich DP, Bankier AA, MacMahon H, et al. Recommendations for the management of subsolid pulmonary nodules detected at CT: A statement from the Fleischner society. Radiology 2013;266:304-17.

31. Detterbeck FC, Marom EM, Arenberg DA, et al. The IASLC lung cancer staging project: Background data and proposals for the application of TNM staging rules to lung cancer presenting as multiple nodules with ground glass or lepidic features or a pneumonic type of involvement in the forthcoming eighth edition of the TNM classification. J Thorac Oncol 2016;11:666-80.

32. Liu M, He WX, Song N, et al. Discrepancy of epidermal growth factor receptor mutation in lung adenocarcinoma presenting as multiple ground-glass opacities. Eur J Cardiothorac Surg 2016;50:909-13.

33. Chung JH, Choe G, Jheon S, et al. Epidermal growth factor receptor mutation and pathologic-radiologic correlation between multiple lung nodules with groundglass opacity differentiates multicentric origin from intrapulmonary spread. J Thorac Oncol 2009;4:1490-5.

34. Li R, Li X, Xue R, et al. Early metastasis detected in patients with multifocal pulmonary ground-glass opacities (GGOs). Thorax 2018;73:290-2.

35. Gaikwad A, Souza CA, Inacio JR, et al. Aerogenous metastases: A potential game changer in the diagnosis and management of primary lung adenocarcinoma. AJR Am J Roentgenol 2014;203:W570-82. 
36. Kadota K, Nitadori J, Sima CS, et al. Tumor spread through air spaces is an important pattern of invasion and impacts the frequency and location of recurrences after limited resection for small stage I lung adenocarcinomas. J Thorac Oncol 2015;10:806-14.

37. Kakinuma R, Noguchi M, Ashizawa K, et al. Natural history of pulmonary subsolid nodules: A prospective multicenter study. J Thorac Oncol 2016;11:1012-28.

38. Kobayashi Y, Fukui T, Ito S, et al. How long should small lung lesions of ground-glass opacity be followed? J Thorac Oncol 2013;8:309-14.

39. Sawada S, Yamashita N, Sugimoto R, et al. Long-term outcomes of patients with ground-glass opacities detected using CT scanning. Chest 2017;151:308-15.

40. Kim HK, Choi YS, Kim J, et al. Management of

Cite this article as: Li M, Wan Y, Zhang L, Zhou LN, Shi Z, Zhang R, Hou YL, Wu N. Synchronous multiple lung cancers presenting as multifocal pure ground glass nodules: are wholebody positron emission tomography/computed tomography and brain enhanced magnetic resonance imaging necessary? Transl Lung Cancer Res 2019;8(5):649-657. doi: 10.21037/ tlcr.2019.09.10 multiple pure ground-glass opacity lesions in patients with bronchioloalveolar carcinoma. J Thorac Oncol 2010;5:206-10.

41. Li M, Wu N, Zhang L, et al. Pathologic N0 status in clinical T1N0M0 lung adenocarcinoma is predictable by the solid component proportion with quantitative CT number analysis. Sci Rep 2017;7:16810.

42. Liu Y, Zhang J, Li L, et al. Genomic heterogeneity of multiple synchronous lung cancer. Nat Commun 2016;7:13200.

43. Saab J, Zia H, Mathew S, et al. Utility of genomic analysis in differentiating synchronous and metachronous lung adenocarcinomas from primary adenocarcinomas with intrapulmonary metastasis. Transl Oncol 2017;10:442-9. 